\title{
Time-resolved brightness measurements by streaking
}

\author{
Joshua S. Torrance, Rory W. Speirs, Andrew J. McCulloch, and Robert E. Scholten \\ School of Physics, The University of Melbourne, Victoria 3010, Australia
}

(Received 30 November 2017; published 29 March 2018)

\begin{abstract}
Brightness is a key figure of merit for charged particle beams, and time-resolved brightness measurements can elucidate the processes involved in beam creation and manipulation. Here we report on a simple, robust, and widely applicable method for the measurement of beam brightness with temporal resolution by streaking one-dimensional pepperpots, and demonstrate the technique to characterize electron bunches produced from a cold-atom electron source. We demonstrate brightness measurements with 145 ps temporal resolution and a minimum resolvable emittance of $40 \mathrm{~nm}$ rad. This technique provides an efficient method of exploring source parameters and will prove useful for examining the efficacy of techniques to counter space-charge expansion, a critical hurdle to achieving single-shot imaging of atomic scale targets.
\end{abstract}

DOI: 10.1103/PhysRevAccelBeams.21.032802

\section{INTRODUCTION}

Charged particle beams are integral to many applications from the Large Hadron Collider at CERN to radiotherapy and other forms of medical treatment [1,2]. The most comprehensive figure of merit for such beams is brightness, which incorporates beam current and emittance [3]. Brightness measurements can also serve as a powerful diagnostic of the processes involved in beam creation and manipulation [4-6]. Time-resolved brightness measurements have the potential to reveal information related to effects such as electron diffusion time, image-charge formation, and space-charge interactions. Here we present a technique for measuring time-resolved brightness by combining pepperpot emittance measurements with beam streaking, and demonstrate the method with electrons generated from a cold-atom electron source (CAES). The technique should be applicable to a wide range of charged particle sources.

The pepperpot method measures the transverse emittance of a charged particle beam using an array of apertures or slits to divide the beam into smaller beamlets that are detected after propagation [7-10]. The divergence, and thus the emittance, can be determined by measuring the position and size of each beamlet, giving an approximation of the transverse phase space of the beam $[9,11]$. The pepperpot method has been used on both electron and ion beams, and with electron energies up to $500 \mathrm{MeV}$ [12].

\footnotetext{
*scholten@unimelb.edu.au
}

Published by the American Physical Society under the terms of the Creative Commons Attribution 4.0 International license. Further distribution of this work must maintain attribution to the author(s) and the published article's title, journal citation, and DOI.
Time-resolved emittance measurements have been performed previously using time-gated detectors to study the feasibility and performance of laser-generated ion sources $[13,14]$, and with scanning slits and the time-resolved signal from a Faraday cup with the aim of minimizing the emittance of an electron storage ring [15]. These methods require many shots to accumulate the temporal profile, and consequently only measure the average beam behavior and are fundamentally incapable of observing shot-to-shot variation in bunch dynamics. Here we outline an alternative method which determines the temporal emittance profile of a bunch by applying a time-varying deflection to the beamlets formed by a pepperpot mask (an array of circular apertures) to "streak" the measurement across the detector. Streaking of electron bunches has been used to observe non-repeatable dynamical processes [16,17], and to characterize the electron pulse itself [18]. With a sufficiently intense source, streak measurements could be performed in a single shot, providing information on shot-toshot behavior to elucidate information unavailable to multiple shot averages, such as transient and stochastic effects.

We demonstrate the technique using electrons from a cold-atom electron source which can provide bunches with diverse pulse duration. Electrons from a CAES are generated by the near-threshold photoionization of laser cooled atoms (see review Ref. [19]). In our experiments, a cloud of rubidium atoms was illuminated by two lasers. The first (red) excites the atoms from the ground state to an intermediate state. The second (blue, pulsed) excites from the intermediate state to either the ionization continuum (above-threshold ionization) or to a high-lying Rydberg state. From the Rydberg state, the atoms could be fieldionized by the static electric field used to accelerate the electrons, or by tunneling across the atomic potential (below-threshold ionization). Previous work has shown 
that electron beams of low temperature, and hence emittance, can be extracted from a CAES and may be capable of producing higher brightness bunches than traditional solid photocathode sources [20]. The streaked-pepperpot technique described here was developed to observe timevarying effects during the photoionization and extraction of electrons from the CAES.

Time-resolved brightness measurements of a CAES can provide information on the effects of techniques to counter space-charge expansion [21], details of the varying ionization processes involved [22], and on beam coherence which is important for potential application to ultrafast electron diffraction (UED). UED imaging and structure determination have the potential to enable measurement of atomiclevel sub-picosecond structural dynamics [23-28]. UED is often performed with photocathode electron sources which have sufficient current and bunch duration for UED but their coherence length of approximately $3 \mathrm{~nm}$ [29], while sufficient for nanocrystalline samples, does not allow diffractive imaging of small biomolecules such as bacteriorhodopsin which has a unit cell length of order $10 \mathrm{~nm}$. The cold atom source has a coherence length of order $10 \mathrm{~nm}$ at the source, without beam expansion, with viable prospects for increasing the current to achieve practical imaging at that scale $[19,20,30]$. Time-resolved knowledge of the source coherence can then be used to improve the image reconstruction of coherent diffractive imaging [31,32].

Electron bunches from the CAES can be produced with durations over a range of timescales, from femtoseconds to microseconds. In our experiments the electron bunch duration was determined by either the ionization rate of the Rydberg state, for below-threshold ionization, or by the pulse length of the laser that coupled the intermediate excited state to the ionization continuum, for above-threshold ionization. With the use of femtosecond duration pulse lasers, a CAES can produce ultrashort electron bunches, which are dense enough to experience space-charge expansion and the related loss of beam quality [21,33,34]. One of the obstacles to single-shot UED is degradation of beam quality due to spacecharge expansion and streaked pepperpot measurements could be used to observe the performance of techniques designed to counter that expansion [21].

The production of electrons from photoionized cold atoms is a complex process, and the temporal profile of pulses from these sources has been characterized under a variety of ionization conditions [22]. The same complex ionization processes that result in variable pulse duration are also likely to affect the transverse velocity of the emitted electrons, and thus the emittance, as a function of time. The streaked pepperpot method presented here allows the transverse velocity spread of the liberated electrons to be measured as a function of time, which can illuminate the underlying atomic ionization processes and provide a diagnostic which could allow optimization of electron bunch brightness in the future.

\section{THEORY}

The normalized transverse brightness of a charged particle beam represents the current density per unit solid angle and for an axially-symmetric beam can be defined as

$$
B_{n, \perp}(t) \equiv \frac{I(t)}{8 \pi^{2} \epsilon_{n, x}^{2}(t)}
$$

where $I(t)$ is the time-varying beam current, and $\epsilon_{n, x}$ is the root-mean-square (RMS) normalized emittance in the plane transverse to the beam propagation axis [3]. The normalized RMS emittance can be defined as

$$
\epsilon_{n, x} \equiv \beta \sqrt{\left\langle x^{2}\right\rangle\left\langle x^{\prime 2}\right\rangle-\left\langle x x^{\prime}\right\rangle^{2}}
$$

where $\beta=v_{z} / c$ is the normalization constant, $v_{z}$ is the velocity along the beam axis, $x$ is the transverse position of an electron and $x^{\prime} \equiv(X-x) / \delta z$ with $X$ the electron position at a later plane separated from the first by a distance along the beam axis $\delta z$. The brackets $\langle\ldots\rangle$ indicate the ensemble average taken over the electron bunch. Equation (2) provides a practical method for calculating emittance using the pepperpot method, where expansion of the pepperpot beamlets is used to determine $x^{\prime}$ [11].

For a thermal source, the normalized RMS emittance of an electron bunch can be estimated from $[3,30]$

$$
\epsilon_{n, x}=\sigma_{x} \sqrt{\frac{k_{B} T}{m_{e} c^{2}}}
$$

where $k_{B}$ is the Boltzmann constant, $\sigma_{x}$ is the RMS width of the source and $T$ is the temperature of the electrons at the source. From Eq. (3) it is apparent that there are two main avenues for reducing the emittance of a source: reducing the source temperature and reducing the size of the source. With a CAES the low source temperature $(T<10 \mathrm{~K})$ can achieve low emittance with moderate source size; $\sigma_{x}=$ $340 \mu \mathrm{m}$ in our case. Low emittance can also be achieved with hot electron sources by using a small source size, for example as small as $\sigma_{x}=1.7 \mu \mathrm{m}$ [35].

Equation (3) is particularly useful to confirm the accuracy of emittance measurements of a CAES because the temperature of the source, and thus the emittance, can be manipulated with the flexible two-color ionization scheme. The transverse temperature at the source can be calculated from

$$
T=\frac{\Delta E}{k_{B}},
$$

where in the absence of an electric field, the excess ionization energy is

$$
\Delta E \approx-E_{I}+\frac{h c}{\lambda_{R}}+\frac{h c}{\lambda_{B}} .
$$


$E_{I}=4.18 \mathrm{meV}$ is the ground state ionization energy of rubidium-85 [36] and $\lambda_{R, B}$ are the wavelengths of the red and blue ionization lasers respectively. Equation (5) gives the approximate excess energy after ionization and is appropriate for the results presented where the contribution to the excess energy from the ionization lasers is greater than the contribution from other effects, for measurements above the resolution limit. A more complete discussion of the ionization process, including the contribution from the accelerating field, can be found in Ref. [37].

\section{SETUP}

In the CAES (Fig. 1) photoionization of a cloud of lasercooled rubidium occurs between two accelerator electrodes, separated by $50 \mathrm{~mm}$. Photoionization was performed with a two-color ionization scheme with a red $780 \mathrm{~nm}$ continuous laser to excite from the ground state to an intermediate state and a wavelength-tunable blue 460-490 nm 5 ns-duration pulse laser was used to excite from the intermediate state directly to the ionization continuum or to a high-lying Rydberg state which, due to the accelerating field, is above the Stark-shifted ionization threshold $[22,38]$.

The electron source operated at the operating frequency of the Q-switched blue pulse laser $(10 \mathrm{~Hz})$. The experimental sequence consisted of the loading of a magnetooptic trap (MOT) for $90 \mathrm{~ms}$, then extinguishing the trapping lasers and magnetic fields $5 \mathrm{~ms}$ before the ionization, such that all atoms returned to the ground state. The accelerator electrodes produced a static electric field of $3.2 \mathrm{kV} \mathrm{cm}^{-1}$ and accelerated the electrons to $8 \mathrm{keV}$. The electrons propagated along the beam line, through the pepperpot mask and onto the phosphor-coupled microchannel plate (MCP) detector.

The transverse spatial profile of each electron bunch was controlled by the Gaussian spatial profile of the red

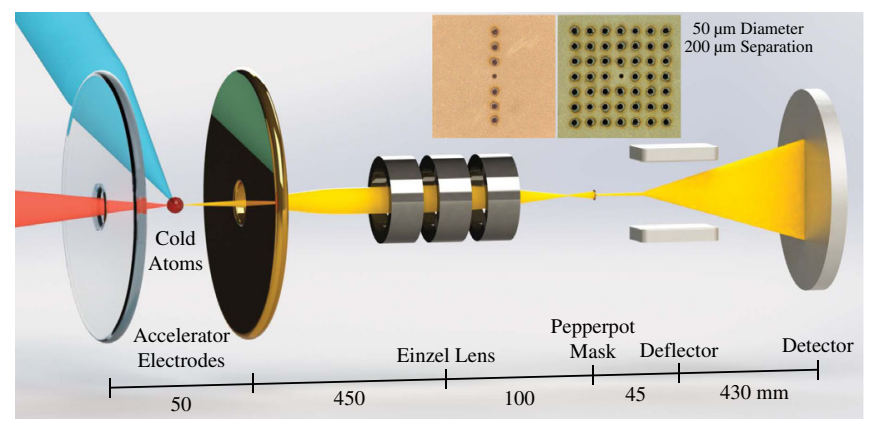

FIG. 1. An overview of the CAES and streaked pepperpot apparatus. Atoms were cooled and trapped in a magneto-optic trap (not shown) before ionization and acceleration along the beamline. Blue and red indicate the two ionization laser beams and yellow indicates the electron beam path. Shown inset are images of the one- and two-dimensional pepperpot masks both with $50 \mu \mathrm{m}$ diameter apertures separated by $200 \mu \mathrm{m}$. ionization laser beam which had an RMS (root mean square) width of $340 \mu \mathrm{m}$ [39]. The Gaussian transverse electron beam profile was confirmed by examining the unobstructed beam incident on the detector as well as the profile of the beamlets after passing through the pepperpot masks.

An Einzel lens was located after the accelerator to focus the beam through a waist and to then expand to the approximate size of the pepperpot mask. The focusing was used to maximize the flux of transmitted electrons and to increase the beam divergence so that beam expansion was measurable at the detector. The sample stage was located $100 \mathrm{~mm}$ after the Einzel lens and was followed by the streak deflector and MCP detector, as shown in Fig. 1.

The deflector was formed by a pair of parallel electrodes, one at ground potential and the other supplied with a timevarying potential synchronized with the experimental cycle, to streak the electron bunches across the detector. Depending on the ionization pathway, bunches were produced on timescales of a few nanoseconds or a few tens of microseconds [22]. To appropriately streak bunches on such timescales two different voltage ramps were used. A bipolar push-pull solid state switch with a fixed transition time of $10 \mathrm{~ns}$ was used for the short bunches. For longer duration bunches the deflector potential was supplied by an amplified voltage ramp supplied by a signal generator with a transition time minimum of $10 \mu \mathrm{s}$.

The dimensions of the pepperpot were constrained by the low temperature of the electrons and upper limit of $2 \mathrm{~mm}$ diameter imposed by the sample holder. An aperture diameter of $50 \mu \mathrm{m}$ was chosen to allow sufficient electron flux in a few hundred shots while resolving emittance as small as $40 \mathrm{~nm}$ rad. An aperture separation of $200 \mu \mathrm{m}$ was selected to provide sufficient bunch sampling for full-bunch profile estimation and manageable beamlet overlap. Oneand two-dimensional pepperpot masks were laser micromachined from $25 \mu \mathrm{m}$ thick, $3 \mathrm{~mm}$ diameter copper disks, producing a single line of seven apertures or a square array of apertures with seven along each edge, as shown in the inset of Fig. 1.

\section{RESULTS}

The wavelength of the blue ionization laser could be adjusted to control the excess ionization energy of the electrons and thus the emittance of the electron beam. If given sufficient energy by the ionization scheme the electrons were excited directly to the ionization continuum producing relatively hot, short-duration electron bunches. If the ionization energy was less than the ionization threshold the electrons were excited to Rydberg states and the atoms field-ionized to produce long-duration cold electron bunches [22]. The predictable change in emittance with excess ionization energy is shown in Fig. 2 for twodimensional pepperpots. Figure 2 validates the pepperpot measurements by comparing the data with the theoretical 


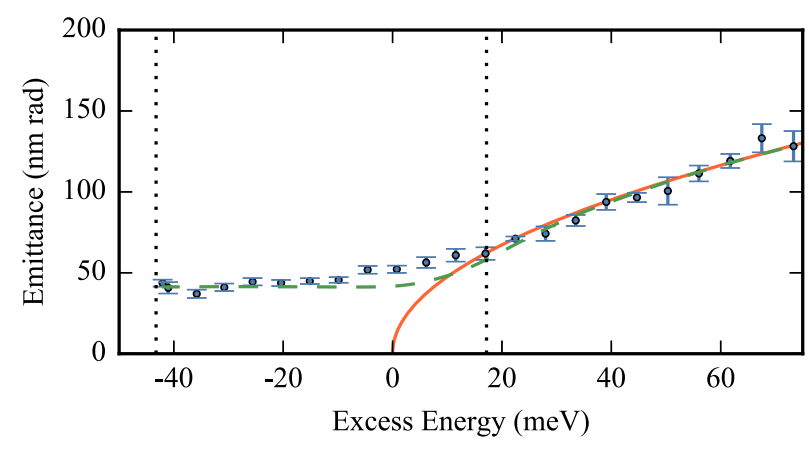

FIG. 2. Electron beam normalized RMS emittance for a range of excess energies for two-dimensional pepperpot measurements (blue circles), analysis of results from a simulation (green dashed line), and calculated using Eq. (3) (solid red line). Excess ionization energy below zero indicates below-threshold fieldionization. The left dotted line indicates the excess energy of the long duration streaked bunch in Fig. 3, and the right is that of the short duration bunch.

prediction of Eq. (3) and the analysis of the results of a particle tracking simulation of the experiment.

Electron bunches produced by field ionization are known to have a duration of order $10 \mu \mathrm{s}$ as the electrons slowly tunnel across the barrier formed by the Stark-shifted Coulomb potential [38], whereas bunches produced from above-threshold direct ionization had a duration determined by the blue ionization laser pulse duration of $5 \mathrm{~ns}$.

The minimum resolvable emittance of the measurement is determined by the size of the apertures used in the pepperpot mask. When the aperture size is such that it makes a non-negligible contribution to the beamlet size, the beamlet size can be corrected by deconvolving the aperture profile from the beamlet profile at the pepperpot plane. Deconvolution is unable to resolve the true divergence in cases where the contribution to the beamlet size by the aperture is greater than that of the divergence and this results in a lower bound on the measurable emittance as shown in Fig. 2. In the measurements presented here a correction has been applied to account for the finite aperture size, with a minimum resolvable emittance of $40 \mathrm{~nm}$ rad. The minimum resolvable emittance could be improved with smaller apertures however, due to the relatively low electron count per bunch for this CAES, the streaked measurement described below would become difficult to resolve with few electrons transmitted through the mask from each bunch.

Pepperpot analysis typically assumes that the beamlets are completely separated, but if there is a limited degree of overlap it is possible to approximate the true size of the beamlets by fitting overlapping beamlet profiles to the data. The data used in these measurements contains a small degree of overlap and the size of the beamlets was determined by fitting the sum of independent Gaussian functions to the data in order to more accurately determine the overlapped beamlet size.
To calculate the brightness of a bunch the beam current must be known. The beam current can be determined from the pepperpot measurements by considering the beam profile sampled by the pepperpot apertures. The number of electrons in each beamlet and the dimensions of the pepperpot mask are known, and given the Gaussian beam shape of the unobstructed beam it is then possible to approximate the profile of the beam without additional measurements, allowing the brightness of the beam to be calculated.

Given the constraints on the pepperpot extent and aperture separation it was not possible to contain the entire electron bunch within the extent of the pepperpots while providing sufficient sampling of the bunch profile to determine the full beam profile. An equipment-specific geometric corrective factor was calculated via simulation and applied during the analysis to account for the truncated emittance measurement.

Figure 3 shows two streaked pepperpot measurements, from 1000-shot averages, used to calculate the temporal brightness profile of the electron bunches. The figure shows two sets of electron bunches, one generated from belowthreshold ionization, resulting in a long-duration bunch and

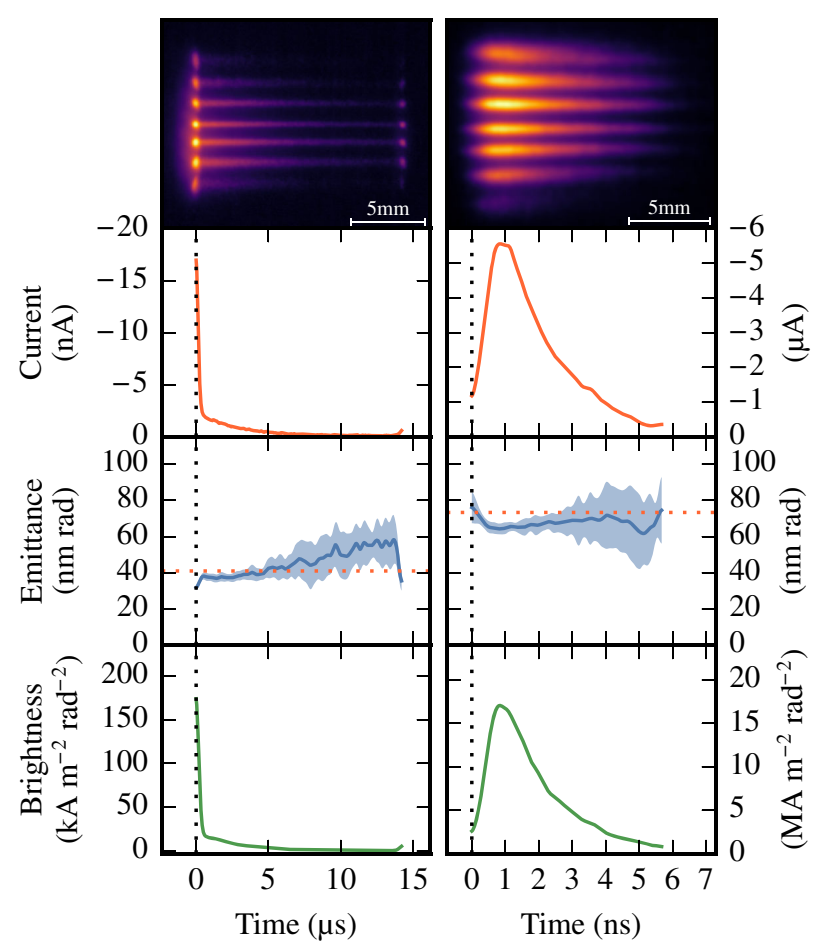

FIG. 3. Long (left) and short (right) duration streaked onedimensional pepperpot measurements. From top to bottom: the false-color 1000-shot average streak image, the full beam current, the normalized RMS emittance of the beam with the shaded region indicating the standard deviation of ten 100-shot subaverages, and the normalized brightness of the beam. The dotted black line indicates the start of the electron bunch and the dotted red lines on the emittance measurements indicate the expected emittance from the simulations shown in Fig. 2. 
the other from above-threshold ionization, resulting in a short-duration bunch. The bunches are streaked across the detector after having passed through the one-dimensional pepperpot mask, with streak times appropriate for the bunch duration. A summary of the parameters of each data set is shown in Table I. Figure 3 shows measurements for long and short bunch durations, controlled using the wavelength of the blue ionization laser. The laser wavelength determines the excess ionization energy and thus whether the ionization is above- or below-threshold, and consequently whether the electron bunches had long or short duration.

The long-duration bunch shows a high bunch current during the first $5 \mathrm{~ns}$ of the bunch, while the blue ionization laser is present, decreasing as the electrons tunnel out of the atomic potential. An apparent increase in current occurs towards the end of the bunch which actually indicates the end of the deflector sweep and atoms continuing to ionize.

The emittance of the two streaked bunches was constant and matches up well with the expected emittance indicated in Fig. 2. One might expect increased bunch emittance during the blue ionization laser pulse due to multiphoton excitation which produces hotter electrons. The results in Fig. 3 indicate that, for these experiments, due to the reduction in the intensity of the blue ionization laser, multiphoton excitation was less significant compared to other measurements performed with this source [22].

For each column of pixels in the streaked measurements, calculations were performed to determine the emittance and full beam profile, and thus brightness, of the beam. Assuming radial symmetry, the beam profile can be extrapolated from the pepperpot data allowing for determination of $I(t)$ for Eq. (1). The long and short duration bunches had a total of $3.6 \times 10^{3}$ and $2.4 \times 10^{3}$ electrons transmitted through the pepperpot per shot. The unobstructed electron bunches contained $8.3 \times 10^{4}$ and

TABLE I. Laser parameters involved in the creation of the bunches and bunch characteristics for the long- and shortduration bunches shown in Fig. 2. The electron count is estimated from the streaked measurements which detected a total of $3.6 \times$ $10^{3}$ and $2.4 \times 10^{3}$ electrons for the long- and short-duration bunches respectively.

\begin{tabular}{lcc}
\hline \hline & Long-duration & Short-duration \\
\hline Red Laser & $780.2 \mathrm{~nm}$ & $780.2 \mathrm{~nm}$ \\
& $\mathrm{CW}$ & $\mathrm{CW}$ \\
Blue Laser & $487.2 \mathrm{~nm}$ & $475.9 \mathrm{~nm}$ \\
& $5 \mathrm{~ns} \mathrm{pulse}$ & $5 \mathrm{~ns} \mathrm{pulse}$ \\
$\Delta E$ & $-43.25 \mathrm{meV}$ & $17.18 \mathrm{meV}$ \\
Ionization Mode & below-threshold & above-threshold \\
Bunch Duration & $\sim 10 \mu \mathrm{s}$ & $\sim 5 \mathrm{~ns}$ \\
Electron Count & $8.3 \times 10^{4}$ & $6.6 \times 10^{4}$ \\
Average $\epsilon_{n, x}$ & $45.6 \mathrm{~nm} \mathrm{rad}$ & $67.6 \mathrm{~nm} \mathrm{rad}$ \\
Peak $B_{n, \perp}$ & $174 \mathrm{kA} \mathrm{m} \mathrm{rad}$ & $17.1 \mathrm{MA} \mathrm{m} \mathrm{rad}$ \\
\hline \hline
\end{tabular}

$6.6 \times 10^{4}$ electrons for the long and short duration bunches, according to the full beam profile estimations. The absolute number of electrons for an image was determined by calibrating the MCP against a Faraday cup with femtoammeter.

To perform these measurements in a single shot with the same parameters and signal-to-noise ratio would require 1000 times more electrons per shot, approximately $10^{8}$, which can be achieved with existing photocathode sources $[16,40]$. The CAES has demonstrated bunches of $10^{6}$ electrons [22], and a number of strategies should enable much larger bunches, such as increasing the density of the MOT, selecting more efficient ionization pathways [41], or the use of a higher intensity blue ionization laser.

The temporal resolution of these measurements was limited by the point spread function of the detector, the transverse size of the beamlets and the slew rate of the deflectors. The long and short bunch duration measurements had full-width-half-maximum temporal resolutions of $308 \mathrm{~ns}$ and $145 \mathrm{ps}$, respectively. The streaking resolution was chosen to show the bunch features on timescales relevant to our bunches, however the same method can be extended to femtosecond ultrafast bunches using rf cavities or photo-activated ultrafast switched voltages which can provide temporal resolution of less than 100 fs $[16,42-44]$.

\section{CONCLUSION}

We have shown the first example of a time-resolved brightness measurement via the streaking of a pepperpot shadow. The technique was developed to observe timevarying effects during the ionization and extraction of electrons from a CAES but the results show that the emittance over the duration of the bunch is constant, indicating that there are no time-dependent effects on source emittance. The emittance for the above-threshold electrons matches predictions from Eq. (3) whereas the below-threshold bunch measurement was resolutionlimited due to the experimental constraints on the pepperpot mask design and the extremely low emittance achievable with a CAES. The next generation of CAES designs will be capable of significantly higher bunch charge, which will make single-shot measurements feasible, allowing for more precise measurements and permitting investigations of temporal bunch behavior in the femtosecond regime. By utilizing ultrafast ionization pathways in a CAES, ultrashort electron bunches can be generated [22,45] and the pepperpot-streaking system shown here could also prove useful in examining the efficacy of techniques to counter space charge expansion by observing the temporal behavior of space-charge driven emittance degradation [21]. This technique could serve as a powerful tool for the determination of time-resolved brightness for charged particle beams. 


\section{ACKNOWLEDGMENTS}

This work was supported by the Australian Research Council through Discovery Project No. DP170101148 and Linkage Project No. LP150101188.

[1] L. Evans and P. Bryant, LHC machine, J. Instrum. 3, S08001 (2008).

[2] S. Verdú-Andrés, U. Amaldi, and Á. Faus-Golfe, Literature review on LINACs and FFAGs for hadron therapy, Int. J. Mod. Phys. A 26, 1659 (2011).

[3] M. Reiser, Theory and Design of Charged Particle Beams (John Wiley \& Sons, New York, 2008).

[4] A. P. Mills Jr., Brightness enhancement of slow positron beams, Appl. Phys. 23, 189 (1980).

[5] J. Qiang, S. Lidia, R. D. Ryne, and C. Limborg-Deprey, Three-dimensional quasistatic model for high brightness beam dynamics simulation, Phys. Rev. ST Accel. Beams 9 , 044204 (2006).

[6] I. V. Bazarov, B. M. Dunham, and C. K. Sinclair, Maximum Achievable Beam Brightness from Photoinjectors, Phys. Rev. Lett. 102, 104801 (2009).

[7] L. E. Collins and P. T. Stroud, Extraction of high current ion beams with low divergence, Nucl. Instrum. Methods 26, 157 (1964).

[8] A. Van Steenbergen, Evaluation of particle beam phase space measurement techniques, Nucl. Instrum. Methods 51, 245 (1967).

[9] J. G. Wang, D. X. Wang, and M. Reiser, Beam emittance measurement by the pepper-pot method, Nucl. Instrum. Methods Phys. Res., Sect. A 307, 190 (1991).

[10] E. Brunetti, R. P. Shanks, G. G. Manahan, M. R. Islam, B. Ersfeld, M. P. Anania, S. Cipiccia, R. C. Issac, G. Raj, G. Vieux, G. H. Welsh, S. M. Wiggins, and D. A. Jaroszynski, Low Emittance, High Brilliance Relativistic Electron Beams from a Laser-Plasma Accelerator, Phys. Rev. Lett. 105, 215007 (2010).

[11] M. Zhang, Emittance Formula for Slits and Pepper-pot Measurement (Fermi National Accelerator Lab., Batavia, 1996), http://lss.fnal.gov/archive/test-tm/1000/fermilabtm-1988.pdf.

[12] N. Delerue, R. Bartolini, K. Peach, A. Reichold, R. Senanayake, S. Bajlekov, L. Caballero-Bendixsen, T. Ibbotson, C. Thomas, Nicolas Bourgeois, B. Buonomo, G. Doucas, S. Hooker, P. Lau, D. Urner, and P. A. Walker, in Proceedings of the 23rd Particle Accelerator Conference, Vancouver, Canada, 2009 (IEEE, Piscataway, NJ, 2009), p. TH5RFP065.

[13] J. Collier, G. Hall, H. Haseroth, H. Kugler, A. Kuttenberger, K. Langbein, R. Scrivens, T. Sherwood, J. Tambini, B. Sharkov, A. Shumshurov, and K. Masek, The CERN laser-ion source, Laser Part. Beams 14, 283 (1996).

[14] M. Yoshida, J. Hasegawa, S. Fukata, Y. Oguri, M. Ogawa, M. Nakajima, K. Horioka, S. Maebara, and M. Shiho, A simple time-resolved emittance measurement of a laser ion source with a digital camera, Nucl. Instrum. Methods Phys. Res., Sect. A 464, 582 (2001).
[15] M. Walter, D. Lamb, S. Bernal, I. Haber, R. A. Kishek, H. Li, B. Quinn, M. Snowel, A. Valfells, M. Reiser, and P. O. O'Shea, in Proceedings of the 20th Particle Accelerator Conference, PAC-2003, Portland, OR, 2003 (IEEE, New York, 2003), Vol. 4, p. 2574.

[16] R. Li, W. Huang, Y. Du, L. Yan, Q. Du, J. Shi, J. Hua, H. Chen, T. Du, H. Xu, and C. Tang, Note: Single-shot continuously time-resolved $\mathrm{MeV}$ ultrafast electron diffraction, Rev. Sci. Instrum. 81, 036110 (2010).

[17] K. Mukojima, S. Kanzaki, K. Kawanishi, K. Sato, and T. Abukawa, Streak-camera reflection high-energy electron diffraction for dynamics of surface crystallography, Surf. Sci. 636, 25 (2015).

[18] D. Ratner, C. Behrens, Y. Ding, Z. Huang, A. Marinelli, T. Maxwell, and F. Zhou, Time-resolved imaging of the microbunching instability and energy spread at the Linac Coherent Light Source, Phys. Rev. ST Accel. Beams 18, 030704 (2015).

[19] A. J. McCulloch, B. M. Sparkes, and R. E. Scholten, Cold electron sources using laser-cooled atoms, J. Phys. B 49, 164004 (2016).

[20] S. D. Saliba, C. T. Putkunz, S. V. Sheludko, A. J. McCulloch, K. A. Nugent, and R.E. Scholten, Spatial coherence of electron bunches extracted from an arbitrarily shaped cold atom electron source, Opt. Express 20, 3967 (2012).

[21] D. J. Thompson, D. Murphy, R. W. Speirs, R. M. W. van Bijnen, A. J. McCulloch, R.E. Scholten, and B. M. Sparkes, Suppression of Emittance Growth Using a Shaped Cold Atom Electron and Ion Source, Phys. Rev. Lett. 117, 193202 (2016).

[22] R. W. Speirs, A. J. McCulloch, B. M. Sparkes, and R.E. Scholten, Identification of competing ionization processes in the generation of ultrafast electron bunches from coldatom electron sources, Phys. Rev. A 95, 053408 (2017).

[23] B. J. Siwick, J. R. Dwyer, R. E. Jordan, and R. J. Dwayne Miller, An atomic-level view of melting using femtosecond electron diffraction, Science 302, 1382 (2003).

[24] C. T. Putkunz, A. J. DAlfonso, A. J. Morgan, M. Weyland, C. Dwyer, L. Bourgeois, J. Etheridge, A. Roberts, R. E. Scholten, K. A. Nugent, and L.J. Allen, Atom-Scale Ptychographic Electron Diffractive Imaging of Boron Nitride Cones, Phys. Rev. Lett. 108, 073901 (2012).

[25] B. M. Sparkes, D. J. Thompson, A. J. McCulloch, D. Murphy, R. W. Speirs, J. S. Torrance, and R. E. Scholten, High-coherence electron and ion bunches from lasercooled atoms, Microsc. Microanal. 20, 1008 (2014).

[26] V. R. Morrison, R. P. Chatelain, K. L. Tiwari, A. Hendaoui, A. Bruhcs, M. Chaker, and B. J. Siwick, A photoinduced metal-like phase of monoclinic $\mathrm{VO}_{2}$ revealed by ultrafast electron diffraction, Science 346, 445 (2014).

[27] M. Gulde, S. Schweda, G. Storeck, M. Maiti, H. K. Yu, A. M. Wodtke, S. Schfer, and C. Ropers, Ultrafast lowenergy electron diffraction in transmission resolves polymer/graphene superstructure dynamics, Science $\mathbf{3 4 5}$, 200 (2014)

[28] S. P. Weathersby et al., Mega-electron-volt ultrafast electron diffraction at SLAC National Accelerator Laboratory, Rev. Sci. Instrum. 86, 073702 (2015). 
[29] T. van Oudheusden, E. F. de Jong, and S. B. van der Geer, Electron source concept for single-shot sub-100 fs electron diffraction in the $100 \mathrm{keV}$ range, J. Appl. Phys. 102, 093501 (2007).

[30] A. J. McCulloch, D. V. Sheludko, M. Junker, and R. E. Scholten, High-coherence picosecond electron bunches from cold atoms, Nat. Commun. 4, 1692 (2013).

[31] S. Flewett, H. M. Quiney, C. Q. Tran, and K. A. Nugent, Extracting coherent modes from partially coherent wavefields, Opt. Lett. 34, 2198 (2009).

[32] B. Abbey, L. W. Whitehead, H. M. Quiney, D. J. Vine, G. A. Cadenazzi, C. A. Henderson, K. A. Nugent, E. Balaur, C. T. Putkunz, A. G. Peele, G. J. Williams, and I. McNulty, Lensless imaging using broadband X-ray sources, Nat. Photonics 5, 420 (2011).

[33] O. J. Luiten, S. B. van der Geer, M. J. de Loos, F. B. Kiewiet, and M. J. van der Wiel, How to Realize Uniform Three-Dimensional Ellipsoidal Electron Bunches, Phys. Rev. Lett. 93, 094802 (2004).

[34] T. van Oudheusden, P. L. E. M. Pasmans, S. B. van der Geer, M. J. de Loos, M. J. van der Wiel, and O. J. Luiten, Compression of Subrelativistic Space-Charge-Dominated Electron Bunches for Single-Shot Femtosecond Electron Diffraction, Phys. Rev. Lett. 105, 264801 (2010).

[35] S. Tokita, S. Inoue, S. Masuno, M. Hashida, and S. Sakabe, Single-shot ultrafast electron diffraction with a laseraccelerated sub-MeV electron pulse, Appl. Phys. Lett. 95, 111911 (2009).

[36] D. A. Steck, Rubidium 85 D Line Data (Oregon Center for Optics and Department of Physics, University of Oregon, 2008), http://steck.us/alkalidata.

[37] W. J. Engelen, E. J. D. Vredenbregt, and O. J. Luiten, Analytical model of an isolated single-atom electron source, Ultramicroscopy 147, 61 (2014).

[38] A. J. McCulloch, R. W. Speirs, J. Grimmel, B. M. Sparkes, D. Comparat, and R. E. Scholten, Field ionization of
Rydberg atoms for high-brightness electron and ion beams, Phys. Rev. A 95, 063845 (2017).

[39] A. J. McCulloch, D. V. Sheludko, S. D. Saliba, S. C. Bell, M. Junker, K. A. Nugent, and R. E. Scholten, Arbitrarily shaped high-coherence electron bunches from cold atoms, Nat. Phys. 7, 785 (2011).

[40] P. Musumeci, J. T. Moody, C. M. Scoby, M. S. Gutierrez, H. A. Bender, and N. S. Wilcox, High quality single shot diffraction patterns using ultrashort megaelectron volt electron beams from a radio frequency photoinjector, Rev. Sci. Instrum. 81, 013306 (2010).

[41] B. M. Sparkes, D. Murphy, R. J. Taylor, R. W. Speirs, A. J. McCulloch, and R.E. Scholten, Stimulated Raman adiabatic passage for improved performance of a coldatom electron and ion source, Phys. Rev. A 94, 023404 (2016).

[42] G. H. Kassier, K. Haupt, N. Erasmus, E. G. Rohwer, H. M. von Bergmann, H. Schwoerer, S. M. M. Coelho, and F. D. Auret, A compact streak camera for 150 fs time resolved measurement of bright pulses in ultrafast electron diffraction, Rev. Sci. Instrum. 81, 105103 (2010).

[43] Y. Ding, C. Behrens, P. Emma, J. Frisch, Z. Huang, H. Loos, P. Krejcik, and M-H. Wang, Femtosecond X-ray pulse temporal characterization in free-electron lasers using a transverse deflector, Phys. Rev. ST Accel. Beams 14, 120701 (2011).

[44] J. F. M. van Rens, W. Verhoeven, J. G. H. Franssen, A. C. Lassise, X. F. D. Stragier, E. R. Kieft, P. H. A. Mutsaers, and O. J. Luiten, Theory and particle tracking simulations of a resonant radiofrequency deflection cavity in $\mathrm{TM}_{110}$ mode for ultrafast electron microscopy, Ultramicroscopy 184, 77 (2018).

[45] R. W. Speirs, C. T. Putkunz, A. J. McCulloch, K. A. Nugent, B. M. Sparkes, and R. E. Scholten, Single-shot electron diffraction using a cold atom electron source, J. Phys. B 48, 214002 (2015). 